\title{
Adanir, Fikret
}

\section{Wandlungen des deutschen Türkeibildes in der ersten Hälfte des 20.} \section{Jahrhunderts}

Lohmann, Ingrid [Hrsg.]; Böttcher, Julika [Hrsg.]: Türken- und Türkeibilder im 19. und 20. Jahrhundert. Pädagogik, Bildungspolitik, Kulturtransfer. Bad Heilbrunn : Verlag Julius Klinkhardt 2021, S. 23-42. - (Wie die Türken in unsere Köpfe kamen. Eine deutsche Bildungsgeschichte; 1)

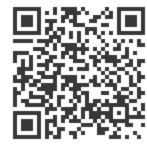

Quellenangabe/ Reference:

Adanir, Fikret: Wandlungen des deutschen Türkeibildes in der ersten Hälfte des 20. Jahrhunderts In: Lohmann, Ingrid [Hrsg.]; Böttcher, Julika [Hrsg.]: Türken- und Türkeibilder im 19. und 20.

Jahrhundert. Pädagogik, Bildungspolitik, Kulturtransfer. Bad Heilbrunn : Verlag Julius Klinkhardt 2021, S. 23-42 - URN: urn:nbn:de:0111-pedocs-219305 - DOI: 10.25656/01:21930

https://nbn-resolving.org/urn:nbn:de:0111-pedocs-219305

https://doi.org/10.25656/01:21930

in Kooperation mit / in cooperation with:

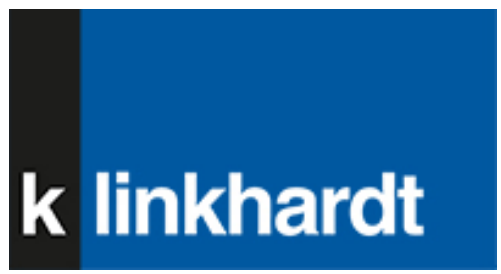

http://www.klinkhardt.de

\section{Nutzungsbedingungen}

Dieses Dokument steht unter folgender Creative Commons-Lizenz: http://creativecommons.org/licenses/by-nc-sa/4.0/deed.de - Sie dürfen das Werk bzw. den Inhalt unter folgenden Bedingungen vervielfältigen, verbreiten und öffentlich zugänglich machen sowie Abwandlungen und Bearbeitungen des Werkes bzw. Inhaltes anfertigen: Sie müssen den Namen des Autors/Rechteinhabers in der von ihm festgelegten Weise nennen. Dieses Werk bzw. der Inhalt darf nicht für kommerzielle Zwecke verwendet werden. Die neu entstandenen Werke bzw. Inhalte dürfen nur unter Verwendung von Lizenzbedingungen weitergegeben werden, die mit denen dieses Lizenzvertrages identisch oder vergleichbar sind.

Mit der Verwendung dieses Dokuments erkennen Sie die Nutzungsbedingungen an.

\section{Terms of use}

This document is published under following Creative Commons-License: http://creativecommons.org/licenses/by-nc-sa/4.0/deed.en - You may copy, distribute and transmit, adapt or exhibit the work in the public and alter, transform or change this work as long as you attribute the work in the manner specified by the author or licensor. You are not allowed to make commercial use of the work. If you alter, transform, or change this work in any way, you may distribute the resulting work only under this or a comparable license.

By using this particular document, you accept the above-stated conditions of use.

\section{Kontakt / Contact:}

\section{peDOCs}

DIPF | Leibniz-Institut für Bildungsforschung und Bildungsinformation Informationszentrum (IZ) Bildung

E-Mail: pedocs@dipf.de

Internet: www.pedocs.de

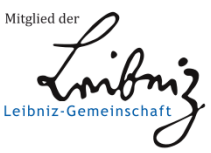




\title{
Wie die Türken in unsere Köpfe kamen. Eine deutsche Bildungsgeschichte
}

\author{
herausgegeben von \\ Ingrid Lohmann, Julika Böttcher, Christine Mayer \\ und Sylvia Kesper-Biermann
}

Band 1

Die Reihe versammelt interdisziplinäre Forschungsergebnisse und Quellentexte zu den deutsch-türkischen Bildungsbeziehungen im 19. und 20. Jahrhundert. Sie nimmt transnationale sowie postkoloniale Perspektiven ein und trägt so zum erweiterten Verständnis eines heute weitgehend unbekannten Kapitels der deutschen Bildungsgeschichte bei. Die Reihe zielt darauf ab, die Historiographie deutscher Pädagogik zu erweitern und das Selbstverständnis der im Bildungswesen Tätigen zu hinterfragen. 
Ingrid Lohmann Julika Böttcher

(Hrsg.)

\section{Türken- und Türkeibilder im 19. und 20. Jahrhundert}

Pädagogik, Bildungspolitik, Kulturtransfer 
Dieser Titel wurde in das Programm des Verlages mittels eines Peer-Review-Verfahrens aufgenommen. Für weitere Informationen siehe www.klinkhardt.de.

Bibliografische Information der Deutschen Nationalbibliothek Die Deutsche Nationalbibliothek verzeichnet diese Publikation in der Deutschen Nationalbibliografie; detaillierte bibliografische Daten sind im Internet abrufbar über http://dnb.d-nb.de.

2021.lg. (C) by Julius Klinkhardt.

Abbildung Umschlagseite 1: Der Schachtürke, nach dem Kupferstich in Joseph Friedrich zu Racknitz: Ueber den Schachspieler des Herrn von Kempelen und dessen Nachbildung. Leipzig: Breitkopf 1789, Tafel III.

Digitale Sammlungen der Humboldt-Universität zu Berlin, urn:nbn:de:kobv:11-d-4709479.

Druck und Bindung: AZ Druck und Datentechnik, Kempten.

Printed in Germany 2021.

Gedruckt auf chlorfrei gebleichtem alterungsbeständigem Papier.

Die Publikation (mit Ausnahme aller Fotos, Grafiken und Abbildungen) ist veröffent-

licht unter der Creative Commons-Lizenz: CC BY-NC-SA 4.0 International

https://creativecommons.org/licenses/by-nc-sa/4.0/

ISBN 978-3-7815-5874-8 digital doi.org/10.35468/5874

ISBN 978-3-7815-2436-1 


\section{Inhaltsverzeichnis}

Ingrid Lohmann, Julika Böttcher

Einleitung und Dank

\section{Diskurse und Wandlungsprozesse}

Fikret Adanır

Wandlungen des deutschen Türkeibildes in der ersten Hälfte

des 20. Jahrhunderts

Ingrid Lohmann

Lieber Türken als Levantiner -

Deutsche Kolonialinteressen an der Türkei und die Stimme

der Pädagogik im langen 19. Jahrhundert

\section{Julika Böttcher}

Die Türkei als Argument in der pädagogischen Presse des

Wilhelminischen Kaiserreichs

\section{Schulbuchanalysen}

Timm Gerd Hellmanzik

„Als Brüder sind die Türken niemals betrachtet, auch der europäischen

Geistesbildung immer fremd geblieben“ - Das Türkenbild in Joseph Annegarns

Weltgeschichte für die katholische Jugend (1840)

Dennis Mathie

„Am schlimmsten erging es den Türken“-

Explorative Untersuchung des Wissens über die Türken und die Türkei

zwischen 1919 und 1936 anhand von drei Schulbuchreihen. 
Andreas Hoffmann-Ocon, Norbert Grube

„Wo die Parteien sich mit Staatsstreichen, Militärrevolten, Hinrichtungen und Morden bekämpfen " - Türkeibilder in schweizerischen

Geschichtslehrmitteln in der ersten Hälfte des 20. Jahrhunderts

\section{Experten und Kulturtransfer}

Filiz Meşeci Giorgetti

Reformpädagogik in türkischen Elementarschulen. Eine Untersuchung zum türkisch-deutschen Austausch über Erziehung und Unterricht in der ersten Hälfte des 20. Jahrhunderts

Christine Mayer

Zur Formierung einer transnationalen Sphäre durch Bildungsexperten John Deweys Reise in die Türkei 1924

\section{Christian Roith}

Pädagogische Modernisierungsstrategien in Spanien

zu Beginn des 20. Jahrhunderts

Sebastian Willert

„Hamdi hat hier gewütet \& da gewühlt“ -

Perspektiven deutscher Forschungsreisender auf die Anfänge der osmanischen Archäologie, 1881-1892

Über die Autorinnen und Autoren .275 
Fikret Adanır

\section{Wandlungen des deutschen Türkeibildes in der ersten Hälfte des 20. Jahrhunderts ${ }^{1}$}

Zusammenfassung: Das deutsche Türkeibild widerspiegelt vornehmlich die auf die Türkei bezogenen Projektionen und Erwartungen in der deutschen öffentlichen Meinung. Wie die gesellschaftlich-politischen Verhältnisse im Allgemeinen ist auch dieses Bild strukturell wie inhaltlich einem epochalen Wandel unterworfen. So zeigten sich die Gebildeten der Wilhelminischen Zeit von der antiken Zivilisation Kleinasiens ebenso tief beeindruckt, wie sie über die gegenwärtigen desolaten Verhältnisse enttäuscht waren. In Unternehmerkreisen war man jedoch zugleich davon überzeugt, dass es eine Leichtigkeit wäre, den verwahrlosten Landschaften der osmanischen Türkei wieder zu ihrer einstigen Blüte zu verhelfen - nur müsse Deutschland bereit sein, seiner "historischen Berufung im Orient" nachzukommen. Eine solche Einstellung, die dem preußisch-deutschen Streben nach Weltgeltung entgegenkam, führte einerseits zu dem grandiosen Bagdadbahnprojekt, andererseits aber in die deutsch-türkische „Waffenbrüderschaft" im Ersten Weltkrieg. Nach der Niederlage von 1918 trat Ernüchterung ein - die imperiale Position im Orient schien für immer verloren. Parallel dazu setzte - nicht zuletzt im Hinblick auf "die Armeniergreuel“ - eine kritische Würdigung des bisherigen Türkei-Engagements ein. Der Erfolg der türkischen Widerstandsbewegung unter der Führung Mustafa Kemals (Atatürk) wiederum fand vor allem in nationalsozialistischen Kreisen der Weimarer Republik Bewunderung und Nachahmung. In der Konstellation des Zweiten Weltkriegs rückte dann auch die Tatsache in den Vordergrund, dass die Türkei über kriegswichtige Rohstoffe wie Chrom, Kupfer und Baumwolle verfügte, deren ungehinderte Zulieferung es zu sichern galt, was die Bewahrung der Neutralität der Türkei voraussetzte. In der frühen Nachkriegszeit entstand vorübergehend ein positives Türkeibild, und zwar dank Beiträge jener deutschen Akademiker und Politiker, die in die Türkei emigriert waren und nun zurückkehrten. Allerdings war dies mit der Bedeutung, die der Türkei im Bewusstsein der Eliten der Wilhelminischen Ära beigemessen wurde, nicht annähernd zu vergleichen.

1 Geringfügig erweiterte und mit Anmerkungen versehene Fassung eines Vortrages, der im Rahmen der türkisch-deutschen Tagung „Geschichte und Kultur der modernen Türkei im Bild der deutschen Öffentlichkeit: Bestandsaufnahme und Strategien zum Abbau von Defiziten“ am 3. Mai 1991 in Ankara gehalten wurde. - Der Beitrag erschien zuerst in der Zeitschrift für Türkeistudien 2 (1991), S. 195-211; er wurde für den vorliegenden Band redaktionell angepasst. 


\section{Einleitung}

Historiker, die sich mit Reisebeschreibungen als Quellengattung befassen, kennen das Problem: Reisebeschreibungen sagen oft mehr über die Heimat der Reisenden als über das fremde Land aus, in dessen Geheimnisse eingedrungen zu sein die Autoren eigentlich glaubten. ${ }^{2}$ Dieser Erfahrungssatz bestätigt sich auch in der beziehungsgeschichtlich-imagologischen Forschung. $\mathrm{Ob}$ es nun um das Russlandbild der Briten um die Zeit des Krimkrieges, das Preußenbild der Franzosen in den Jahrzehnten vor der deutschen Reichsgründung oder das französische Türkenbild in der Zeit des nationalen Widerstandes in Anatolien gegen das Versailler System geht: Die im eigenen Land dominierenden Interessen und die vielfältigen Wünsche, Befürchtungen und sonstigen Vorstellungen der Bevölkerung erweisen sich als die bestimmenden Faktoren. ${ }^{3}$ Auf unser Thema bezogen bedeutet dies, dass das deutsche Türkeibild im 20. Jahrhundert in erster Linie die Struktur und Dynamik der öffentlichen Meinung in Deutschland selbst reflektiert und viel weniger die Zustände in der Türkei.

Der Begriff „öffentliche Meinung“ ist allerdings, vor allem in Bezug auf seine empirische Fassbarkeit, problematisch. Als Ausdruck des Gesamtwillens eines ganzen Volkes hat es sie wohl nie gegeben. ${ }^{4}$ Im Zusammenhang mit dem deutschen Türkeibild ist demnach zu betonen, dass man eher von vielfaltigen Vorstellungen von der Türkei auszugehen hat, welche bei verschiedenen Personen, Interessengruppen, Schichten und Klassen in einem gegebenen Zeitraum vorgeherrscht haben. Uns interessieren diese Vorstellungen, insofern sie beziehungsgeschichtlich relevant geworden sind, das heisst, wirtschaftlich, politisch und kulturell auf das deutsch-türkische Verhältnis eingewirkt haben. Unter diesen Prämissen lassen sich die Wandlungen des deutschen Türkeibildes im 20. Jahrhundert sinnvoll periodisieren und in ihren einzelnen Phasen darstellen.

2 Vgl. Harbsmeier, Michael: Reisebeschreibungen als mentalitätsgeschichtliche Quellen. Überlegungen zu einer historisch-anthropologischen Untersuchung frühneuzeitlicher deutscher Reisebeschreibungen. In: Reiseberichte als Quellen europäischer Kulturgeschichte. Hrsg. von Antoni Mączak, Hans-Jürgen Teuteberg. Wolfenbüttel: Herzog-August Bibliothek 1982, S. 1-31.

3 Vgl. Krautheim, Hans-Jobst: Öffentliche Meinung und imperiale Politik. Das britische Russlandbild 1815-1854. Berlin: Duncker \& Humblot in Kommission 1977; Wenger, Klaus Rudolf: Preussen in der öffentlichen Meinung Frankreichs 1815-1870. Politische Aspekte des französischen Preußenbildes. Ein Beitrag zur historischen Analyse nationaler Urteilsklischees. Göttingen: Musterschmidt 1979; Akyüz, Yahya: Türk Kurtuluş Savaşı ve Fransız kamuoyu (1919-1922). 2., erw. Aufl. Ankara: Türk Tarih Kurumu 1988.

4 Vgl. Skalweit, Stephan: Frankreich und Friedrich der Große. Bonn: Röhrscheid 1952, S. 1, zitiert in Krautheim: Öffentliche Meinung, S. 21. 


\section{Deutsche Türkeibilder zu Beginn des 20. Jahrhunderts}

Das wilhelminische Deutschland war im Bewusstsein der Zeitgenossen eine verspätete Großmacht. Gegenüber seinen Rivalen hatte Deutschland besonders im Bereich der imperialistischen Expansion noch einiges nachzuholen. Die Protagonisten der deutschen Großmachtpolitik waren sich indes darüber im Klaren, dass ihnen angesichts der gespannten Lage in Europa nicht viel Zeit zur Realisierung grandioser Pläne bleiben würde. Gegen Ende des 19. Jahrhunderts war das Deutsche Reich zunehmend in außenpolitische Isolierung geraten. Nicht nur gegenüber Frankreich, das von Rachegelüsten wegen der Niederlage von 1871 geleitet war, musste man sich in Acht nehmen. Auch zu Russland und, was noch schwerer wog, zu Großbritannien, der Führungsmacht der Epoche, gestalteten sich die Beziehungen konfliktträchtig. Diese Konstellation warf ihren Schatten auch auf die langfristigen Entwicklungsaussichten der deutschen Wirtschaft. Die Stimmen mehrten sich, die eine Neuorientierung in den Außenbeziehungen des Reiches forderten. In einer Schrift, die seinerzeit viel Beachtung fand, heißt es:

„Gegenüber dem immer engeren Abschluss Amerikas und Rußlands gegen europäische Manufakte und dem drohenden Zusammenschluss Englands und seiner Kolonien zu einer wirtschaftlichen Einheit erscheint für das Deutsche Reich die einzig richtige Politik die der Handelsverträge mit dem außerrussischen Europa und Asien und die schließliche Erweiterung derselben zu einem eigentlichen Zollbunde."

Wie könnte das Deutsche Reich angesichts solch widriger Umstände als gleichberechtigter Partner etwa eines Großbritanniens noch Weltgeltung erlangen? Im Grunde ging es um diese Frage - für das wilhelminische Deutschland hatte die Türkei ihren Stellenwert in diesem Kontext. Das türkische Reich lag nämlich in derjenigen Weltregion, in welche wirtschaftlich und politisch zu expandieren für Deutschland noch möglich zu sein schien. Die Entwicklung der deutsch-türkischen Beziehungen seit dem Beginn des 20. Jahrhunderts ist in dieser Perspektive zu betrachten. Zunächst im militärischen Bereich kam es zu einer engen deutsch-türkischen Zusammenarbeit. Deutsche übernahmen Funktionen in der osmanischen Armee, während osmanische Offiziere turnusgemäß nach Deutschland entsandt wurden, um sich in der preußischen Armee zu spezialisieren. Die Bedeutung dieser neuen Beziehung wurde durch die Orientbesuche Kaiser Wilhelms II. beim Sultan-Kalifen in Istanbul $(1889,1898)$ unterstrichen. Den politischen Initiativen folgten bald solche im wirtschaftlichen Gebiet, und mit dem Bagdadbahnprojekt befand man sich schon auf dem Weg zur „deutsch-türkischen Waffenbrüderschaft“ im Weltkrieg.

5 Kaerger, Karl: Kleinasien. Ein deutsches Kolonisationsfeld. Kolonialwirthschaftliche Studie. Berlin: Gergonne 1892, S. 91. 
Woran dachten die Deutschen konkret beim Wort „Türkei“? Das komplexe Türkeibild der Deutschen zu Beginn des 20. Jahrhunderts lässt sich für unseren Zweck auf einige wenige Punkte reduzieren. Als erstes verdient die Einstellung des deutschen Bildungsbürgertums zur Türkei Berücksichtigung. Innerhalb dieser Schicht lässt sich seit Mitte des 19. Jahrhunderts eine besondere Empfindsamkeit für die vergangene Größe der orientalischen Zivilisation feststellen. Im Mittelpunkt des Interesses stand das Kleinasien der Antike, d.h. die Vergangenheit des Kernlandes des Osmanischen Reiches. Die Archäologie vermittelte in diesem Zusammenhang ein Bild von einem ehemals märchenhaft reichen und dichtbevölkerten Land. In der Gegenwart allerdings befand sich Kleinasien offensichtlich in einem völlig verwahrlosten Zustand, in weiten Strecken wüst und menschenleer. Die Enttäuschung des Europäers begann schon in Thrakien: „Dem Reisenden, der, von Westund Mitteleuropa kommend, mit der Eisenbahn nach Konstantinopel fährt, tritt die traurige Lage der Bodenkultur sofort vor Augen [...]. Kaum ist die Grenze überschritten, so wird er [...] auf der langen Strecke von Adrianopel bis zur Hauptstadt von der Armseligkeit des Anbaues [...] peinlich berührt. "6 Diese scheinbare Öde war jedoch andererseits keineswegs ein Endzustand. Denn, eine adäquate Bewirtschaftung vorausgesetzt, war es eine Leichtigkeit, den nahöstlichen Landschaften wieder zu ihrer einstigen Blüte zu verhelfen. Nur müsste Deutschland bereit sein, seiner historischen Berufung im Orient nachzukommen. ${ }^{7}$

Die hier zunächst nur anklingende Idee der Kolonisation rückte bald in den Bereich des politisch Machbaren. Es waren vor allem „liberale Imperialisten“ wie Paul Rohrbach und Friedrich Naumann, professionelle „Orientkenner“ wie Paul Dehn und Hugo Grothe, aber auch der Chef der deutschen Militärmission in Istanbul, Colmar Freiherr von der Goltz, die sich zu Wort meldeten, um Wege und Mittel einer neuen Türkeipolitik in kolonialistischer Perspektive zu empfehlen. Im Jahre 1901 entstand die Deutsch-Asiatische Gesellschaft, 1905 wurde die Deutsche Vorderasien-Gesellschaft gegründet. Neue Schriftenreihen wie Beiträge zur Kenntnis des Orients, Länder und Völker der Türkei, Der neue Orient, Deutsches Vorderasien- und Balkanarchiv und andere fanden ihr Publikum. ${ }^{8}$

Die Fertigstellung der Anatolischen Bahn (lzmit - Ankara 1892, Eskişehir - Afyon 1895), die man stolz als „die deutsche Bahn“ apostrophierte, und vor allem die Erteilung der Konzession zum Bau der weiterführenden Strecken bis nach Bagdad (1899/1903) ebneten den Weg zu einer Welle von Kleinasien-Reisenden, die überaus eifrig Material über Land und Leute sammelten, um sie einer wissbe-

6 Morawitz, Karl: Aus Arbeitstagen und Mußestunden. Wien: Braumüller 1907, S. $54 f$.

7 Vgl. Kampen, Wilhelm van: Studien zur deutschen Türkeipolitik in der Zeit Wilhelms II. (Diss.) Kiel: Disputats 1968, S. 84-88.

8 Vgl. Schöllgen, Gregor: „Dann müssen wir uns aber Mesopotamien sichern!“ Motive deutscher Türkenpolitik zur Zeit Wilhelms II. in zeitgenössischen Darstellungen. Saeculum 32 (1981), S. 130-145, hier $132 f$. 
gierigen Öffentlichkeit in der Heimat zu präsentieren: Nachgezeichnet wurde das Bild eines Landes, das reich an Bodenschätzen war, die unter Einsatz deutscher Ingenieursbegabung mit geringen Investitionsmitteln zu Tage zu fördern wären. Auch das agrarische Produktionspotential wurde als sehr groß eingeschätzt. Es stand außer Frage, dass Anatolien sich eines Tages zur Kornkammer Europas entwickeln würde.

Die Bewohner des Landes bildeten, so die zeitgenössischen deutschen Darstellungen, ein buntes Völkergemisch. Immer wieder wurde auf die dominierende Stellung der Muslime im Staatsdienst und in der Landwirtschaft hingewiesen. Im Allgemeinen war der Muslim „ungeschickter, plumper“ und vermochte „im Wettbewerbe mit den Andersgläubigen nur mit geringem Erfolge zu bestehen." Aus diesem Grunde lägen die „mit dem Handel verknüpften oder verwandten Berufsarten größtenteils oder fast ausschließlich in den Händen der unterdrückten Bevölkerungsgruppen. "9 $\mathrm{Zu}$ diesen Berufsarten zählte man Bankiers und Geldwechsler, Handelsagenten, Dolmetscher und Fremdenführer, Zeitungsverleger, Ärzte und Rechtsanwälte. Was könnte Deutschland mit einem solchen „Menschenmaterial“ anfangen? Max Freiherr von Oppenheim war in dieser Hinsicht nicht pessimistisch. Das Einzugsgebiet der Bagdadbahn vor Augen, verglich er das Völkergemisch im Osmanischen Reich mit jenem in den Vereinigten Staaten von Amerika und kam dabei zu der Schlussfolgerung: „Wenn nun die einzelnen Menschenrassen [...] in kultureller Beziehung fraglos weniger wertvoll sind als das amerikanische Volk, ist doch jede einzelne derselben weit besser als der amerikanische Neger, gar nicht zu reden vom Indianer. ${ }^{\text {"10 }}$ Seiner Meinung nach sollte Deutschland eine Zusammenarbeit in erster Linie mit dem muslimischen Element anstreben, denn die „eingeborenen Christen“ waren „übertrieben geldgierig, unwahrheitsliebend, intrigant und dabei eitel und feige". Er hielt das Wort des großen Moltke immer noch für gültig, nämlich, „dass der einzige Gentleman im Orient der Türke, d.h. der Mohammedaner"11 sei.

Für Friedrich Naumann, um einen ebenfalls sehr einflussreichen Zeitgenossen zu zitieren, spielte bei dieser Präferenz des muslimischen Elements auch die weltpolitische Konkurrenzsituation zu England eine wichtige Rolle. Mit deutlicher Anspielung auf die Massaker an Armeniern in den 1890er Jahren konnte Naumann sogar empfehlen, gegenüber dem Leiden „der christlichen Völker im türkischen

9 Lichtenstädter, Siegfried: Nationalität, Religion und Berufsgliederung im Oriente. In: Jahrbuch der Münchner Orientalischen Gesellschaft. Hrsg. von Hugo Grothe. (Beiträge zur Kenntnis des Orients VIII) Halle an der Saale: Gebauer-Schwetschke 1910, S. 42-70, hier 62-64.

10 Oppenheim, Max Freiherr von: Zur Entwickelung des Bagdadbahngebietes und insbesondere Syriens und Mesopotamiens unter Nutzanwendung amerikanischer Erfahrungen. Berlin: Liebheit \& Theisen 1904, S. 154.

11 Ebd. S. 177. 
Reiche politisch gleichgültig"12 zu bleiben, und zwar weil England sich für diese einsetzte, mit dem Ziel, die Türkei zu schwächen. Die Türkei war aber gegenüber Großbritannien zu unterstützen. Nur so könnte sie Schauplatz künftiger deutscher Großmachtpolitik bleiben: „Wir müssen das Land wirtschaftlich von uns abhängig machen, um es später politisch ,kontrollieren' zu können. “13 Sollte dies nicht gelingen, so stand für Friedrich Naumann auch das fernere Ziel fest: „Selbst wenn wir Konstantinopel nicht für uns brauchen können, wollen wir an der Konkursmasse des Osmanischen Reiches beteiligt sein." ${ }^{14}$

Das wilhelminische Deutschland war trotzdem davon überzeugt, dass die wirtschaftliche und politische Durchdringung der Türkei nicht zuletzt im Interesse der Türken selbst lag. Diese Überzeugung kommt in der Frage einer deutschen Kolonisation Kleinasiens deutlich zum Vorschein. Dabei hatten die Anhänger der Kolonisationsidee vor, gerade die schönsten und fruchtbarsten Gebiete Kleinasiens zu besiedeln, so etwa die „Gegend des Sebandja-Sees, wo die Berghänge herrliche Lagen für den Weinbau geben, während die Ebene ein vorzügliches Feld für Maulbeer-Olivenzucht bietet", oder die Landschaften am mittleren Lauf des Sakarya-Flusses, weil sich hier "noch weite Waldgebiete vor(finden), schöne Berge, fruchtbare Thäler wie in Deutschland. " ${ }^{15}$ Sie erwarteten, dass man den Kolonisten, die ihre deutsche Staatsangehörigkeit sollten beibehalten dürfen, unentgeltlich ein zusammenhängendes Territorium überließ. Die deutschen Gemeinden auf diesem Territorium sollten Autonomie genießen, mit eigener Gerichtsbarkeit, mit einer eigenen deutschen Polizei. Diese Gemeinden sollten für zehn Jahre keine Steuern zahlen und danach lediglich eine für alle Zeiten fixierte Pauschsumme. Die zollfreie Einfuhr bestimmter Gegenstände sollte ihnen ebenso gewährt werden wie die „Erlaubnis zur Ausprägung größerer mit der steigenden Einwanderung wachsender Summen von Kleingeld." ${ }^{16}$

Eine derartige Kolonisation Anatoliens - statt Auswanderung von Millionen von Deutschen nach Übersee - hätte für den deutschen Staat große Vorteile gehabt. Der wichtigste davon war, dass die Ausgewanderten in ihrer neuen Heimat nicht assimiliert werden würden. Denn „der Staat hat [...] doch das allergrößte Interesse da-

12 Naumann, Friedrich: „Asia“. Eine Orientreise über Athen, Konstantinopel, Baalbek, Nazareth, Jerusalem, Kairo, Neapel. Berlin: Buchverlag der „Hilfe“ 1899, S. 144f, zitiert in Schöllgen: „Dann müssen wir uns aber Mesopotamien sichern!“, S. 135.

13 Naumann, Friedrich: „Asia“. Eine Orientreise über Athen, Konstantinopel, Baalbek, Nazareth, Jerusalem, Kairo, Neapel. 7. Aufl. Berlin: Buchverlag der „Hilfe“ 1909, S. 164.

14 Ebd. S. 34.

15 Schlagintweit, Max: Deutsche Kolonisationsbestrebungen in Kleinasien. Vortrag gehalten in der Deutschen Kolonial-Gesellschaft, Abteilung Berlin, am 8. Januar 1900. München: Wolf \& Sohn 1900, S. 33f.

16 Kaerger: Kleinasien, S. 90. Der kolonialistische Wunschkatalog Kaergers wurde von gleichgesinnten Autoren oft unverändert übernommen, so von Schlagintweit: Deutsche Kolonisationsbestrebungen, S. 46. 
ran, dass ihm die Auswanderer, der Überschuß seiner Bevölkerung, nicht verloren gehen, dass sie auch im Auslande ihre volkstümlichen Sitten und Anschauungen beibehalten und in wirtschaftlicher Verbindung mit dem Mutterlande bleiben ". ${ }^{17}$ Aber auch dem Osmanischen Reich würden daraus nicht minder bedeutende Vorteile erwachsen: „Ein Paar gesetzgeberische Maßregeln zu Gunsten deutscher Einwanderer, nur einige willensstarke, europäisch gebildete Beamte mit eigenen Machtbefugnissen, und eine blühende Kultur würde sich in diesen Ländern wieder wie in alter Zeit entwickeln und den türkischen Staat sicherer schützen als das Beharren in überlebten Zuständen." ${ }^{18}$

Man muss allerdings hinzufügen, dass nicht alle „Kolonialisten“ auf die kleinasiatische Karte setzten. Gewichtige Stimmen hielten es für klüger, die deutsche Auswanderung weiterhin nach Übersee, z.B. nach Südwestafrika, zu lenken statt nach Kleinasien, wo es ohnehin fraglich erschien, ob die deutschen Bauern „den schon infolge der Blutrache unausbleiblichen, immer erneuten Angriffen der umwohnenden kriegerischen Völker gegenüber sich würden halten können." ${ }^{19}$ Ein einflussreicher „Kolonialist“ wie Hans Delbrück plädierte gar für die baltische Option. Seiner Meinung nach sollte Deutschland bestrebt sein, die Einwilligung Russlands für eine deutsche Expansion in Richtung Baltikum zu gewinnen, und dafür bereit sein, russische Südexpansion auf Kosten des Osmanischen Reiches zu akzeptieren. ${ }^{20}$ Es waren vor allem die deutschen Kapitalisten, angeführt vom Direktor der Deutschen Bank Georg von Siemens, die den Plan einer deutschen Kolonisation in Kleinasien für ein Phantasiegebilde hielten. Die Unternehmer der wilhelminischen Ära erwiesen sich durchweg als nüchtern denkende Geschäftsleute mit einem gewissen Sinn für das real Machbare. Nicht einmal für die Bagdadbahn waren sie anfänglich zu begeistern gewesen, sie hatten sich in jenes Abenteuer erst nach reichlichem Druck von oben eingelassen. ${ }^{21}$ Freilich waren sie dann durchaus bestrebt, daraus den größtmöglichen Gewinn zu ziehen, sie waren bestrebt, wie Georg von Siemens es gegen die Auffassungen von Max Schlagintweit formulierte, „durch Aufschließung der dortigen Landesteile und durch Herstellung kommerzieller Verbindungen unserer Industrie und unseren Technikern Beschäftigung und Verdienst, und auf diese Weise unserem Vaterland Vorteil und

17 Ebd. S. 50.

18 Menz, Reinhold: Deutsche Arbeit in Kleinasien. Reiseskizze und Wirthschaftsstudie. Berlin: Springer 1893, zitiert in ebd. S. 35.

19 Oppenheim: Zur Entwickelung des Bagdadbahngebietes, S. $156 f$.

20 Vgl. Sullivan, Charles Donald: Stamboul Crossings. German Diplomacy in Turkey, 1908 to 1914. (PhD thesis) Nashville, Tennessee: Vanderbilt University 1977, S. 21.

21 Vgl. dazu Mejcher, Helmut: Die Bagdadbahn als Instrument deutschen wirtschaftlichen Einflusses im Osmanischen Reich. Geschichte und Gesellschaft 1 (1975), S. 447-481. 
Einfluß ${ }^{\text {“22 }}$ zu verschaffen. Nüchtern blieben auch die deutschen Militärs. Der Generalstab ging von einem künftigen Zweifrontenkrieg um Deutschland aus. Das Osmanische Reich als Bündnispartner - etwa gegen Russland - würde in diesem Falle einen gewissen Flankenschutz für deutsche Truppen z.B. in der Ukraine bedeuten, aber mehr nicht. Vor allem würde Deutschland selbst nie genug Truppen haben, um sich in großangelegten Operationen im Nahen Osten zu engagieren. So war es ratsam, eine zurückhaltende Politik im Orient zu verfolgen, damit man nicht unnötigerweise in undurchschaubare Dinge verwickelt wurde. ${ }^{23}$

\section{Entwicklungen seit der jungtürkischen Revolution}

Die jungtürkische Revolution von 1908 markierte eine gewisse Zäsur in der Entwicklung der deutsch-türkischen Beziehungen. Deutschland hatte mit der Regierung Abdulhamids eng zusammengearbeitet und galt deswegen in den Augen der Jungtürken anfänglich als eine Stütze der Despotie. Die konservativen deutschen Staatsmänner ihrerseits hielten von den überwiegend westlich liberal inspirierten und deshalb zum Kaiserreich auf Distanz gehenden Jungtürken nicht viel. Während in den interessierten Kreisen das Für und Wider des hamidischen Systems debattiert wurde, trat eine merkliche Abkühlung in den Beziehungen zum Osmanischen Reich ein. ${ }^{24}$ In der folgenden Zeit (bis zum Ausbruch des Weltkrieges) schien Berlin in allen die Türkei betreffenden Fragen eine mehr oder weniger antitürkische Stellung einzunehmen: So unterstützte Deutschland im Jahre 1908 die Donaumonarchie, als diese die völkerrechtlich noch immer dem Osmanischen Reich gehörenden Provinzen Bosnien und die Herzegowina annektierte; Deutschland gewährte Italien diplomatische Rückendeckung, als die römische Regierung im Jahre 1911 die osmanische Provinz Tripolis in Nordafrika besetzen ließ.

Anlässlich des Balkankrieges von 1912/13 erfuhr das Verhältnis zur Türkei eine noch stärkere Belastung. Die deutsche Öffentlichkeit wünschte zwar überwiegend einen Erfolg der osmanischen Waffen, die übrigens fast ausschließlich deutscher Herkunft waren, wie auch die osmanische Armeeführung von deutschen Offizieren beraten wurde. Gerade deshalb aber war die Verärgerung besonders groß, als infolge des kläglichen Versagens des türkischen Heeres weltweit der Eindruck

22 Bemerkungen des Herrn von Siemens, in Schlagintweit: Deutsche Kolonisationsbestrebungen, S. 57.

23 Vgl. Sullivan: Stamboul Crossings, S. 15.

24 Vgl. zur Apologie der Politik Abdulhamids: Ular (Uhlemann), Alexander; Insabato, Enrico: Der erlöschende Halbmond. Türkische Enthüllungen. Frankfurt am Main: Rütten \& Loening 1909, und als Antwort darauf Jäckh, Ernst: Der aufsteigende Halbmond. Beiträge zur türkischen Renaissance. Berlin: Fortschritt (Buchverlag der „Hilfe“) 1909, 5., erw. Aufl. Stuttgart: Deutsche Verlags-Anstalt 1915. 
entstand, als würden die deutsche Waffentechnik und die deutsche Kriegskunst wenig taugen. Während der Kämpfe an der Çatalca-Linie, wo das Schicksal der osmanischen Hauptstadt auf des Messers Schneide stand, gab Kaiser Wilhelm selbst dieser Enttäuschung Ausdruck: „Die Türkei in Europa ist zu Ende, sie muss hinaus und je weniger sie Schwierigkeiten macht um so besser. ${ }^{\text {" } 25}$ Deutschland erwartete die baldige Aufteilung auch des verbliebenen osmanischen Besitzes in Asien und war entschlossen, dabei nicht leer auszugehen: „Also Achtung, aufgepaßt, daß die Aufteilung nicht ohne uns gemacht wird“26 - so wies Kaiser Wilhelm seine Minister und Generäle an. Diplomaten wie Botschafter Wangenheim und „Türkenfreunde“ wie der Publizist Ernst Jäckh konnten sich jedoch vorerst dahingehend durchsetzen, dass es für Deutschland vorteilhafter sei, „die Türkei solange wie möglich zu erhalten [...]. Gleichzeitig aber hätten wir uns auf den schlimmsten Fall, die Teilung, vorzubereiten “. ${ }^{27}$ Im griechisch-türkischen Konflikt wegen der Besitzrechte über die ostägäischen Inseln im Jahre 1914 sollte das Deutsche Reich jedenfalls Partei für Griechenland ergreifen. Beim Ausbruch des Weltkrieges im Sommer 1914 maß man in Deutschland der Türkei zunächst kaum Bedeutung bei. Die deutschen Militärs schienen nicht viel von einem Bündnis mit dem Osmanischen Reich zu halten. Man war allgemein der Ansicht, dass die osmanische Armee eher eine Last denn eine Hilfe für die deutsche Kriegsführung sein würde. Zwar war dann der Kriegseintritt des Osmanischen Reiches im Herbst 1914, nachdem der erhoffte deutsche Blitzsieg sich als eine Illusion erwiesen hatte, höchst willkommen. Dennoch sollte nicht übersehen werden, dass trotz der glänzenden Verteidigungsleistung an den Dardanellen der türkische Kriegsbeitrag insgesamt als enttäuschend empfunden wurde. Denn die aus deutscher Sicht wichtigste Trumpfkarte des Sultans, der „Heilige Krieg“, den man als ideologische Waffe zur Revolutionierung der Muslime in den Kolonien gegen die Entente einzusetzen hoffte, erwies sich als unwirksam. Das war gleichsam ein „Verrat“ an Deutschland, wo man doch seit der zweiten Orientreise des Kaisers an den von Abdulhamid sorgfältig gepflegten Mythos geglaubt hatte, dass nämlich die osmanischen Sultane als Kalifen großes Ansehen in der islamischen Welt genössen und durch Ausrufung eines Heiligen Krieges die Entente-Mächte

25 Telegraphische Weisung an Reichskanzler vom 15. November 1912, No. 10, A. 20263. Politisches Archiv des Auswärtigen Amtes, Bonn, Türkei 203, Bd. 8, zitiert in Adanır, Fikret: Deutschland im Spiegel türkischer Geschichtsschreibung. In: Fremdheit und Angst. Beiträge zum Verhältnis von Christentum und Islam. Hrsg. von Doron Kiesel, Şener Sargut, Rosi Wolf-Almanasreh. Frankfurt am Main: Haag und Herchen 1988, S. 159-173, hier 168.

26 Zitiert in Fischer, Fritz: Krieg der Illusionen. Die deutsche Politik von 1911-1914. 2. Aufl. Düsseldorf: Droste 1970, S. 429.

27 Aus einem Bericht Wangenheims vom August 1913, zitiert in ebd. S. 431; vgl. Jäckh, Ernst: Deutschland im Orient nach dem Balkankrieg. München: Mörike 1913. 
an ,weichen' Stellen ihres Herrschaftssystems, etwa am Suez-Kanal oder in Indien, würden treffen können. ${ }^{28}$

Für weitere Ernüchterung sorgten die Jungtürken mit ihrem überraschend selbstbewussten Auftreten gegenüber dem deutschen Bündnispartner im Weltkrieg. Hatten die Deutschen noch im Balkankrieg das Fehlen von Nationalbewusstsein als eine der Ursachen des türkischen Debakels diagnostiziert, so sahen sie sich jetzt einer jungtürkischen Führung gegenüber, die durchaus in der Lage war, eigene nationalistische Initiativen zu entwickeln, ja sogar mit dem deutschen Imperialismus zu konkurrieren. Der spätere Außenminister Baron Neurath, Anfang 1916 Botschaftsrat in Istanbul, soll nach der Einnahme Erzurums durch die Russen einem österreichischen Kollegen gegenüber bezeichnenderweise erklärt haben, man hoffe deutscherseits, dass die schlimme Lage in Ostanatolien „die Türken im allgemeinen kleinmütiger und willfähriger machen "29 werde. Gegen Ende des Weltkrieges kam es im Kaukasus sogar fast zu einem offenen deutsch-türkischen Zusammenstoß. ${ }^{30}$ Trotzdem muss aber betont werden, dass während des Weltkrieges in der deutschen Öffentlichkeit von offizieller Seite ebenso wie von den einschlägigen Verbänden das Bild von einer deutsch-türkischen Schicksalsgemeinschaft lanciert und mit beträchtlichem Erfolg gefördert wurde. ${ }^{31}$ Neben politischen Propagandaschriften erschienen zahlreiche Informationshefte, die die Sachkenntnis in Deutschland vor allem über die wirtschaftlichen und rechtlichen Verhältnisse im Osmanischen Reich zu vermehren halfen. ${ }^{32}$ Unter Beteiligung

28 Zur panislamistischen Propaganda während des Krieges vgl. Hagen, Gottfried: Die Türkei im Ersten Weltkrieg. Flugblätter und Flugschriften in arabischer, persischer und osmanisch-türkischer Sprache aus einer Sammlung der Universitätsbibliothek Heidelberg. Frankfurt am Main: Peter Lang 1990; Fischer, Fritz: Griff nach der Weltmacht. Die Kriegszielpolitik des kaiserlichen Deutschland 1914/18. (Sonderausgabe) Düsseldorf: Droste 1967, S. 113-116.

29 Bihl, Wolfdieter: Die Kaukasus-Politik der Mittelmächte. Teil I: Ihre Basis in der Orientpolitik und ihre Aktionen 1914-1917. Wien: Böhlau 1975, S. 206.

30 Vgl. Baumgart, Winfried: Deutsche Ostpolitik 1918: Von Brest-Litovsk bis zum Ende des Ersten Weltkrieges. Wien: Oldenbourg 1966, S. 299; ders.: Das „Kaspi-Unternehmen“ - Größenwahn Ludendorffs oder Routineplanung des deutschen Generalstabs. Jahrbücher für Geschichte Osteuropas 18 (1970), S. 47-126, 231-278, bes. 81-85. Vgl. auch Trumpener, Ulrich: Germany and the Ottoman Empire, 1914-1918. Princeton, NJ: Princeton University Press 1968, S. 167-199.

31 Vgl. zum Beispiel Becker, Carl Heinrich: Deutsch-türkische Interessengemeinschaft. (Bonner Vaterländische Reden und Vorträge während des Krieges). Bonn: Cohen 1914; Jäckh, Ernst: Die deutsch-türkische Waffenbrüderschaft. In: Der Deutsche Krieg. Politische Flugschriften 24. Hrsg. von Ernst Jäckh. Stuttgart: Deutsche Verlags-Anstalt 1915.

32 Beispielhaft seien folgende Titel genannt: Der Handel der Türkei. Balkan-Revue 2 (1915-1916), S. 111-119, 248-261, 419-428; Vincenz, Friedrich von: Die türkische Tabakregie. Balkan-Revue 1 (1914-1915), S. 118-126; Honig, N. von: Über Industrie und Handwerk in Konstantinopel. Archiv für Wirtschaftsforschung im Orient 1 (1916), S. 421-448, 2 (1917), S. 76-108; Bruck, Werner Friedrich: Vorläufiger Bericht über Baumwoll-Erzeugung und -Verbrauch der Türkei. Augsburg: Liebheit \& Thiesen 1917 (Arbeitsausschuss der Deutschen Baumwollspinnerverbände); Trott-Helge, Else: Die Erweiterung der Tätigkeit der türkischen Landwirtschaftsbank. 
führender Finanziers und Unternehmer des Landes - darunter Arthur von Gwinner, Hjalmar Schacht, Albert Ballin, Franz Johannes Günther, Heinrich Roetger - wurde in Berlin eine Deutsch-Türkische Vereinigung gegründet, mit dem Ziel, die Förderung des deutschen Türkei-Engagements zu koordinieren. ${ }^{33}$

Neben der Tatsache, dass nunmehr Informationen über die Verhältnisse in der Türkei reichlich flossen, waren es vor allem die vor Ort gemachten Erfahrungen deutscher Offiziere, Ingenieure und Kaufleute, die zu einem signifikanten Wandel des deutschen Türkeibildes während der Kriegsjahre führten: Man begann, dem muslimischen Element im Osmanischen Reich Organisationstalent, Energie, ja unternehmerische Fähigkeiten zuzuschreiben..$^{34}$ Dies erforderte aber deutscherseits die Bereitschaft, sich die Zusammenarbeit mit dem türkischen Reich in der Nachkriegszeit auf einer völlig neuen Ebene vorzustellen: Die Türkei würde zwar weiterhin ein wichtiger Rohstofflieferant für Deutschland bleiben. Berücksichtigt wurde aber jetzt auch die Tatsache, dass in diesem Land inzwischen eine einheimische Industrie im Entstehen begriffen war, und man nahm davon Notiz, dass immer mehr Angehörige der türkischen Oberschicht sich in unternehmerischen Bereichen betätigten. So können wir in einer Schrift aus dem Kriegsjahr 1917 gleichsam eine Vorwegnahme des heutigen Entwicklungskonzeptes des Westens für die ,Dritte Welt' entdecken:

„Die Möglichkeit, daß neue Industriezweige in der Türkei sich entwickeln, muß ins Auge gefaßt werden. Eine industriell aufstrebende Türkei ist kaufkräftiger, aufnahmefähiger für unsere Erzeugnisse und wertvoller für unsern Handel [...] als das in dem alten Zustand beharrende Land. ${ }^{\text {"35 }}$

Balkan-Revue 4 (1917-1918), S. 384-387; Seiger, Bernhard: Die Mühlenindustrie in und um Konstantinopel. Balkan-Revue 4 (1917-1918), S. 520-528; Mygind, Eduard: Anatolien und seine wirtschaftliche Bedeutung. Balkan-Revue 4 (1917-1918), S. 1-6; Herlt, Gustav: Die Industrialisierung der Türkei. In: Geld, Industrialisierung und Petroleumschätze der Türkei. Hrsg. von Hugo Grothe et al. Berlin: De Gruyter 1918, S. 41-80.

33 Schopp, Georg Michael: Türkische Jugend in Deutschland. Ein Beitrag zur Geschichte der deutsch-türkischen Beziehungen zu Beginn des 20. Jahrhunderts. In: Düsseldorfer Jahrbuch 74. Hrsg. von Düsseldorfer Geschichtsverein. Düsseldorf: Klartext 2003 (ursprüngliche Referenz: unveröffentlichtes Manuskript).

34 „Der heutige Jungtürke strebt nach vorwärts und wird neben der Reorganisation des Staates auch die Reorganisation des einzelnen Betriebes mit Erfolg anstreben“. Marré, Ernst C.N.: Die Türken und Wir nach dem Kriege. Ein praktisches Wirtschaftsprogramm. Berlin: C.A. Schwetschke \& Sohn 1916, S. 6.

35 Oppenheim, Max Freiherr von: Die Nachrichtensaal-Organisation und die wirtschaftliche Propaganda in der Türkei, ihre Übernahme durch den Deutschen Überseedienst. Berlin: Reichsdruckerei 1917, S. 10. 


\section{Türkeibilder in der Weimarer Republik}

Die Niederlage im Weltkrieg machte freilich die glänzenden Entwicklungsperspektiven auf der Grundlage der deutsch-türkischen Zusammenarbeit im Orient vorerst zunichte. Wie in einer Schrift aus dem Jahre 1919 festgestellt wurde, waren „die ungeheuern Kapitalien, die deutsche Unternehmer in der Türkei investiert" hatten, „wahrscheinlich rettungslos verloren“. In tiefem Pessimismus sagte man dann voraus: „Der Plan der wirtschaftlichen Durchdringung der Türkei ist damit für Deutschland erledigt, man gebe sich nicht den Illusionen hin, dass die Entente es uns jemals wieder gestatten wird, von neuem im Orient Fuß zu fassen. " ${ }^{36}$ Vor allem in linken Blättern wie Die Rote Fahne, Vorwärts oder Die Freiheit konnte man nun eine kritische Würdigung des deutschen Türkei-Engagements, auch im Hinblick auf „die Armeniergreuel“, verfolgen. Die Veröffentlichung im Jahre 1919 von Aktenstücken zur deutschen Haltung in der armenischen Frage war in diesem Zusammenhang ebenso eine Sensation wie die Publikation des stenographischen Berichts über die Verhandlungen im „Prozeß Talaat Pascha“im Jahre $1921 .^{37}$

Die Unabhängigkeitsbewegung in Anatolien wurde unter diesen Bedingungen vor allem in der linken Presse mit zwiespältigen Gefühlen kommentiert. Einerseits war man über die Zukunft christlicher Minderheiten in der Türkei besorgt, andererseits aber - im Sinne Lenins - erfreut über den anti-imperialistischen Kampf der Völker des Ostens. ${ }^{38}$ Die Freude über die Erfolge der Kemalisten stellte sich aber erst richtig in den deutsch-nationalen Kreisen ein, und zwar nicht nur aus Sympathie für das türkische Volk, sondern auch aus Schadenfreude darüber, dass das verhasste Versailler System so bald und ausgerechnet von der Türkei in Frage gestellt wurde. Die Türkei Mustafa Kemal Atatürks sollte in der Zwischenkriegszeit für viele Deutsche das revisionistische Prinzip par excellence verkörpern. In dieser Atmosphäre begannen die alten Asienkämpfer, sich mit Vorliebe

36 Schwarzhaupt, Paul: Die Wahrheit über die Türkei. Die Armeniergreuel und Deutschenbedrückungen durch die Jungtürken. (Revolutions-Bibliothek Nr. 6) Berlin: Gesellschaft und Erziehung 1919, S. 4.

37 Vgl. Lepsius, Johannes: Deutschland und Armenien, 1914-1918. Sammlung diplomatischer Aktenstücke. Potsdam: Tempelverlag 1919; Der Prozess Talaat Pascha. Stenographischer Bericht über die Verhandlung gegen den des Mordes an Talaat Pascha angeklagten armenischen Studenten Salomon Teilirian. Mit einem Vorwort von Armin T. Wegner und einem Anhang. Berlin: Deutsche Verlagsgesellschaft für Politik und Geschichte 1921.

38 Zur Behandlung türkischer Themen in der deutschen linken Presse jener Jahre vgl. die Beiträge von Tunçay, Mete: Alman sol basınında Türkiye konuları. Tarih ve Toplum No. 48 (Dezember 1988), S. 6-8; No. 50 (Februar 1988), S. 5-8; No. 55 (Juli 1988), S. 6-9; 49 No. 56 (August 1988), S. 6-8; No. 57 (September 1988), S. 51-52. 
an die „germanischen Tugenden“"39 der Türken im Weltkrieg zu erinnern. Die Veröffentlichungen in der Weimarer Republik zur Geschichte und Gegenwart der deutsch-türkischen Beziehungen umfassten schon ein breites Spektrum. Die Mehrheit der Titel war der Gattung Memoirenliteratur zuzurechnen und hatte überwiegend entweder politisch-apologetischen oder militärhistorischen Charakter. ${ }^{40}$ Daneben erschienen zahlreiche Studien, die den Transformationsprozess in Staat und Gesellschaft der Türkei thematisierten. ${ }^{41}$ Mustafa Kemal selbst ließ seine große Rede von 1927 in Leipzig drucken, und bald folgten auch solche Publikationen, die sich mit Person und Werk des Führers der neuen Türkei befassten. ${ }^{42}$ Auch der Wirtschaft in der Weimarer Republik musste die Entstehung der Republik Türkei wie ein Glück im Unglück erscheinen. Man hatte im Orient zwar schon viel verloren, aber die Abschaffung der Kapitulationen im Lausanner Frieden bedeutete für Deutschland immerhin eine Gleichstellung mit den Siegermächten auf dem türkischen Markt. ${ }^{43}$ Entsprechend erfolgreich verlief die Wiederanknüpfung der Beziehungen in den 1920er Jahren. In der Weltwirtschaftskrise schließlich geriet die Türkei, wie die übrigen südosteuropäischen Länder,

39 Schlegel, Dietrich: Das Bild der Türkei in der deutschen Presse 1924-1933. Südosteuropa-Mitteilungen 26 (1986), S. 20-39, hier 37.

40 Vgl. Sanders, Liman von: Fünf Jahre Türkei. Berlin: August Scherl 1919; Cemal Paşa, Ahmed: Erinnerungen eines türkischen Staatsmannes. München: Drei Masken 1922; Izzet Pascha, Ahmed: Denkwürdigkeiten. Ein kritischer Beitrag zur Kriegsschuldfrage. Hrsg. von Karl Klinghardt. Leipzig: Koehler 1927; Kannengiesser, Hans: Gallipoli. Bedeutung und Verlauf der Kämpfe 1915. Berlin: Schlieffen 1927; Pomiankowski, Josef: Der Zusammenbruch des ottomanischen Reiches. Erinnerungen an die Türkei aus der Zeit des Weltkrieges. Zürich: Amalthea 1928; Mühlmann, Carl: Der Kampf um die Dardanellen 1915. Oldenburg in Oldenburg: Stalling 1927; ders.: Deutschland und die Türkei 1913-1914. Die Berufung der deutschen Militärmission nach der Türkei 1913, das deutsch-türkische Bündnis 1914 und der Eintritt der Türkei in den Weltkrieg. Berlin-Grunewald: Rothschild 1929; Range, Paul: Vier Jahre Kampf ums Heilige Land. Bad Oeynhausen: Meyer 1932.

41 Vgl. etwa Jäschke, Gotthard: Die Türkei seit dem Weltkriege. Geschichtskalender, 1918-1928. Berlin: Deutsche Gesellschaft für Islamkunde 1929; Ziemke, Kurt: Die neue Türkei - Politische Entwicklung 1914-1929. Berlin: Deutsche Verlags-Anstalt 1930; Hartmann, Richard: Ziya Gökalp's Grundlagen des türkischen Nationalismus. Orientalische Literatur-Zeitung 28 (1925), S. 578-610; Wittek, Paul: Konstantinopel, Islam und Kalifat. Archiv für Sozialwissenschaft und Sozialpolitik 53 (1925), S. 370-426; Rühl, Phillip: Die Türkische Republik in Wirtschaft und Aufbau. (Schriften des Frankfurter Messamts 16) Frankfurt am Main: R. Th. Hauser \& Co. [1925].

42 Vgl. Die neue Türkei 1919-1927. Rede gehalten von Gazi Mustafa Kemal Pascha in Angora vom 15.-20. Oktober 1927 vor den Abgeordneten und Delegierten der Republikanischen Volkspartei. 3 Bde. Leipzig: Koehler 1928; Mikusch, Dagobert von: Gasi Mustafa Kemal. Leipzig: Paul List 1929.

43 Vgl. Fleury, Antoine: La pénétration allemande au Moyen-Orient 1919-1939: Le cas de la Turquie, de l'Iran et de l'Afghanistan. Genève: A.W. Sijthoff 1977, S. 99. - Deutschland hatte ja auf die aus den „Kapitulationen“ herrührenden Privilegien schon zu Beginn des Krieges verzichtet. 
immer stärker in den Sog des deutschen Marktes, auf dem man seine Agrarüberschüsse eher absetzen konnte als in England oder Frankreich, die bessere Produkte und wesentlich billiger aus ihren Kolonien bezogen. ${ }^{44}$

\section{Entwicklungen ab 1933}

Mit dem Aufstieg der NSDAP trat kein signifikanter Wandel in der Türkeieinschätzung der Deutschen ein. Gewiss rief die erfolgreiche Außenpolitik der kemalistischen Republik Respekt in Berlin hervor. In Südosteuropa zum Beispiel würde Deutschland fest mit der Türkei als Faktor zu rechnen haben. Die Neugestaltung der Beziehungen Ankaras zu den einzelnen Balkanstaaten sowie dessen besonderes Verhältnis zur Sowjetunion wurden dabei zunehmend auch unter geopolitischen Gesichtspunkten kommentiert. Von Zentraleuropa aus schien Ankara „zu einem sehr beachtlichen Nebenzentrum der großen Politik“" ${ }^{45}$ geworden zu sein.

Auch im Nahen Osten war die Türkei aus deutscher Sicht immer noch ein „Schlüsselland“. Man ging davon aus, dass sie mit ihrem nationalen Formierungsprozess exemplarische Bedeutung für die anderen Völker der Region hatte. In einer schon 1933 entstandenen Denkschrift, die unter dem Titel Raumpolitik - Kulturpolitik geeignete Schritte zur erfolgreichen Propaganda-Arbeit im Orient vorschlug, wurde die Türkei jedenfalls der künftigen Einflusszone des Dritten Reiches zugerechnet. ${ }^{46}$ Man beabsichtigte, die Zusammenarbeit mit ihr besonders im kulturellen Bereich zu vertiefen. In diesem Rahmen war es wohl nicht ohne Bedeutung, dass die autoritären Züge des türkischen Regimes sowie die Rolle Mustafa Kemals als der unumstrittene Führer des türkischen Volkes in ideologischer Hinsicht die Annahme gewisser Affinitäten zwischen dem türkischen System einerseits und dem italienischen und deutschen Faschismus andererseits nahelegten. ${ }^{47}$

44 Zur Lage der Türkei in der Weltwirtschaftskrise vgl. zuletzt Dawletschin-Lindner, Camilla: Die Türkei in der Weltwirtschaftskrise. In: Der Nahe Osten in der Zwischenkriegszeit 1919-1939. Hrsg. von Linda Schatkowski Schilcher, Claus Scharf. Stuttgart: Franz Steiner 1989, S. 222-240; dies.: Die Türkei und Ägypten in der Weltwirtschaftskrise 1929-1933. Stuttgart: Franz Steiner 1989.

45 Gross, Hermann: Die türkische Republik und Europa. Zeitschrift für Geopolitik II (1934), S. 92f; vgl. ferner Bischoff, Norbert von: Ankara - Eine Deutung des neuen Werdens in der Türkei. Wien: Holzhausen/Bruckmann 1935; Jäschke, Gotthard: Der Weg zur russisch-türkischen Freundschaft. Die Welt des Islams 16 (1934 ), S. 23-38; ders.: Kommunismus und Islam im türkischen Befreiungskriege. Die Welt des Islams 20 (1938), S. 110-117.

46 Zur Information über diese Denkschrift vgl. Tarih ve Toplum 67 (Temmuz 1989), S. 59-61; Fleury: La pénétration allemande, S. $57 \mathrm{ff}$.

47 Vgl. Zapp, Manfred: Türkischer Nationalsozialismus. Preussische Jahrbücher (Aug. 1933), S. 105-112. Allerdings stellte Melzigs Biographie Mustafa Kemal eher als einen Humanisten dar, vgl. Melzig, Herbert: Kamál Atatürk - Untergang und Aufstieg der Türkei. Frankfurt am Main: 
Das Interesse an der Türkei stieg in der Vorbereitungsphase des Zweiten Weltkrieges naturgemäß weiter an. Neben der geostrategischen Lage des Landes rückte nun die Tatsache immer mehr in den Vordergrund, dass die Türkei über einige kriegswichtige Rohstoffe wie Chrom, Kupfer und Baumwolle verfügte. Die Regierung in Ankara blieb jedoch in dieser Hinsicht weit hinter den deutschen Erwartungen zurück, worüber sich die gleichgeschalteten Medien im Dritten Reich immer wieder empörten. Von besonderem Gewicht in diesem Zusammenhang waren natürlich die Ansichten Hitlers. Mustafa Kemal Atatürk soll er schon bewundert haben, von dessen Nachfolger, İsmet İnönü, hielt er aber offensichtlich nicht viel. In seiner Rede vom 22. August 1939 vor den Oberbefehlshabern der Wehrmacht soll er gesagt haben: „Seit dem Tode Kemals wird die Türkei von kleinen Geistern regiert, haltlose, schwache Menschen. " ${ }^{48}$ Der Beistandspakt Ankaras mit den Westmächten vom 19. Oktober 1939 gab daher Anlass zu scharfen Kommentaren in der deutschen Presse. Die Reichsregierung war nunmehr entschlossen, mit allen Mitteln zu verhindern, dass die Türkei auf westlicher Seite in den Krieg eintrat. Die sogenannte Russlandklausel in dem genannten Beistandspakt erwies sich dabei als ein geeignetes Instrument. Mit jener Klausel hatte Ankara einen Kriegseintritt auf Seiten der Westmächte für den Fall ausgeschlossen, wenn dies zur Verschlechterung der Beziehungen zur Sowjetunion hätte führen können. Um nun die Spannungen zwischen Moskau und Ankara künstlich anzuheizen in der Erwartung, dass dies die Türkei eher zur Neutralität verpflichten würde -, war Berlin jedes Mittel recht. Ein Coup in diesem Propagandakrieg, der das Türkeibild besonders in den linken Kreisen negativ beeinflusste, war die Veröffentlichung einer Auswahl französischer Geheimakten, die man während des Frankreichfeldzuges im Juni 1940 erbeutet hatte. Das publizierte Material erweckte den Eindruck, als bereite sich die Türkei im Bunde mit den Westmächten für eine Offensive in Richtung auf die Erdölfelder von Baku vor. Deutscherseits hatte man dabei einige Dokumente absichtlich verfälscht: War zum Beispiel in den Texten einmal von einem „Defensivkrieg“ die Rede, so hatte man daraus einen „Offensivkrieg“" gemacht. ${ }^{49}$

Societäts-Verlag 1937. - Über Auseinandersetzungen und Berührungspunkte des Kemalismus mit dem Faschismus vgl. Carretto, Giacomo E.: Polemiche fra kemalismo, fascismo, communismo negli anni „30“. Storia Contemporanea 8 (1977), S. 489-530; Adanır, Fikret: Zur „Etatismus“Diskussion in der Türkei in der Weltwirtschaftskrise. Die Zeitschrift Kadro 1932-1934. In: Der Nahe Osten in der Zwischenkriegszeit. Die Interdependenz von Politik, Wirtschaft und Ideologie. Hrsg. von Linda Schatkowski Schilcher, Claus Scharf. Stuttgart: Franz Steiner 1989, S. 355-373. 48 Zitiert in Schreiber, Gerhard; Stegemann, Bernd; Vogel, Detlef: Der Mittelmeerraum und Südosteuropa. Das Deutsche Reich und der Zweite Weltkrieg 3. Hrsg. vom Militärgeschichtlichen Forschungsamt. Stuttgart: Deutsche Verlagsanstalt 1984, S. 139.

49 Vgl. ebd. S. $143 f$. 
Zu einer Krise in den deutsch-türkischen Beziehungen kam es im Frühjahr 1941, als die Wehrmacht auf dem Balkan, d.h. im Vorfeld Istanbuls aufmarschierte. In einem persönlichen Handschreiben gelang es jedoch Hitler, Präsident İnönü davon zu überzeugen, dass das Dritte Reich keine kriegerischen Absichten gegenüber der Türkei hege. Erst recht der Beginn des Russlandfeldzuges leitete dann eine neue Annäherung zwischen Berlin und Ankara ein. Die Medien auf beiden Seiten erinnerten nun mit Nachdruck an die alte deutsch-türkische Waffenbrüderschaft. In Deutschland wuchs allmählich die Hoffnung, die Türkei im Rahmen eines panislamistisch-pantürkischen Projekts eventuell doch in den Krieg gegen die Sowjetunion hineinzuziehen. Der diesbezügliche Druck auf die Türkei war im Jahre 1942 vielleicht am größten, als die Deutschen den Terek-Fluss im Kaukasus erreichten. ${ }^{50}$

\section{Schluss}

Die Nachkriegszeit bildet wieder eine neue Phase in der Geschichte der deutschen Türkei-Wahrnehmung. In den ersten Jahren war man vorrangig mit der Beseitigung der Kriegsfolgen beschäftigt, und die weit im südöstlichen Horizont liegende Türkei konnte in diesem Stadium kaum Aufmerksamkeit in der deutschen öffentlichen Meinung beanspruchen. Jene deutschen Wissenschaftler, die im Dritten Reich Zuflucht in der Türkei gefunden hatten und nun sukzessive nach Deutschland zurückkehrten, trugen in gewissem Sinne sicherlich zur Verbreitung eines positiven Türkeibildes bei. ${ }^{51}$ Man darf jedoch die Wirkung dieser Emigranten auf die deutsche öffentliche Meinung nicht überbewerten. Wie Christian Petry anlässlich der Publizierung des Scurla-Berichts (1987) ${ }^{52}$ feststellte, „ist das Ausmaß und die Bedeutung der Migration deutscher Wissenschaftler in die

50 Bezeichnenderweise wurde im April 1942 eine Fotoausstellung in der deutschen Botschaft in Ankara über die deutschen Offiziere, die je in osmanisch-türkischen Diensten gestanden hatten, organisiert. Dieses Ereignis fiel außerdem mit den Feierlichkeiten anlässlich des 100. Geburtstages des Feldmarschalls von der Goltz zusammen. Vgl. Koçak, Cemil: Türkiye’de bir Alman subayı. Nazi Almanya’sının Ankara Ataşemiliteri (1936-44) General H. Rohde'nin Türkiye anıları hakkında oğlu Dr. Dieter Rohde ile bir konuşma. Tarih ve Toplum 5 (Mai 1984), S. 18-27.

51 Zur Emigration deutscher Akademiker in die Türkei vgl. Widmann, Horst: Exil und Bildungshilfe. Die deutschsprachige Emigration in die Türkei nach 1933. Bern: Peter Lang 1973.

52 Vgl. Der Scurla-Bericht. Bericht des Oberregierungsrates Dr. rer. pol. Herbert Scurla von der Auslandsabteilung des Reichserziehungsministeriums in Berlin über seine Dienstreise nach Ankara und Istanbul vom 11. - 25. Mai 1939: „Die Tätigkeit deutscher Hochschullehrer an türkischen wissenschaftlichen Hochschulen“. Hrsg. von Klaus-Detlev Grothusen unter Mitwirkung von Ruşen Keleş. Frankfurt am Main: Dağyeli 1987. 
Türkei“ - trotz der Veröffentlichungen von Fritz Neumark, ${ }^{53}$ Ernst E. Hirsch ${ }^{54}$ und anderen - ,in der Bundesrepublik Deutschland weitgehend unbekannt geblieben." 55

Abschließend kann festgehalten werden, dass die Bedeutung, welche die Türkei im deutschen öffentlichen Bewusstsein während der Wilhelminischen Ära hatte, seither nie mehr erreicht worden ist. Deutschland wagte damals über eine als unabdingbar erachtete wirtschaftlich-politische Durchdringung des Osmanischen Reiches - wie Fritz Fischer es formulierte - den "Griff zur Weltmacht“. Eine vergleichbare Konstellation hat sich nie wieder eingestellt. Das Dritte Reich suchte seinen „Lebensraum“ anderswo, ihm blieb Anatolien peripher. Für die Bundesrepublik Deutschland schließlich, ein Produkt des Kalten Krieges, war die Türkei bloß ein Bündnispartner der zweiten Reihe, genauso instrumentalisierbar wie sie selbst im Spiel der Supermächte.

\section{Quellen und Literatur}

Adanır, Fikret: Deutschland im Spiegel türkischer Geschichtsschreibung. In: Fremdheit und Angst. Beiträge zum Verhältnis von Christentum und Islam. Hrsg. von Doron Kiesel, Şener Sargut, Rosi Wolf-Almanasreh. Frankfurt am Main: Haag und Herchen 1988, S. 159-173.

Adanır, Fikret: Zur „Etatismus“-Diskussion in der Türkei in der Weltwirtschaftskrise. Die Zeitschrift Kadro 1932-1934. In: Der Nahe Osten in der Zwischenkriegszeit. Die Interdependenz von Politik, Wirtschaft und Ideologie. Hrsg. von Linda Schatkowski Schilcher, Claus Scharf. Stuttgart: Franz Steiner 1989, S. 355-373.

Akyüz, Yahya: Türk Kurtuluş Savaşı ve Fransız kamuoyu (1919-1922). 2., erw. Aufl. Ankara: Türk Tarih Kurumu 1988.

Baumgart, Winfried: Deutsche Ostpolitik 1918: Von Brest-Litovsk bis zum Ende des Ersten Weltkrieges. Wien: Oldenbourg 1966.

Baumgart, Winfried: Das „Kaspi-Unternehmen“ - Größenwahn Ludendorffs oder Routineplanung des deutschen Generalstabs. Jahrbücher für Geschichte Osteuropas 18 (1970), S. 47-126, 231278.

Becker, Carl Heinrich: Deutsch-türkische Interessengemeinschaft. (Bonner Vaterländische Reden und Vorträge während des Krieges). Bonn: Cohen 1914.

Bihl, Wolfdieter: Die Kaukasus-Politik der Mittelmächte. Teil I: Ihre Basis in der Orientpolitik und ihre Aktionen 1914-1917. Wien: Böhlau 1975.

Bischoff, Norbert von: Ankara - Eine Deutung des neuen Werdens in der Türkei. Wien: Holzhausen/ Bruckmann 1935.

Bruck, Werner Friedrich: Vorläufiger Bericht über Baumwoll-Erzeugung und -Verbrauch der Türkei. (Arbeitsausschuss der Deutschen Baumwollspinnerverbände). Augsburg: Liebheit \& Thiesen 1917.

53 Vgl. Neumark, Fritz: Zuflucht am Bosporus. Deutsche Gelehrte, Politiker und Künstler in der Emigration 1933-1953. Frankfurt am Main: Knecht 1980.

54 Vgl. Hirsch, Ernst E.: Aus des Kaisers Zeiten durch die Weimarer Republik in das Land Atatürks. München: Schweitzer 1982.

55 Grothusen, in: Der Scurla Bericht, S. 9. 


\section{0| Filkret Adanur}

Carretto, Giacomo E.: Polemiche fra kemalismo, fascismo, communismo negli anni „30“.Storia Contemporanea 8 (1977), S. 489-530.

Cemal Paşa (Djemal Pascha), Ahmed: Erinnerungen eines türkischen Staatsmannes. München: Drei Masken 1922.

Dawletschin-Lindner, Camilla: Die Türkei in der Weltwirtschaftskrise. In: Der Nahe Osten in der Zwischenkriegszeit 1919-1939. Hrsg. von Linda Schatkowski Schilcher, Claus Scharf. Stuttgart: Franz Steiner 1989, S. 222-240.

Dawletschin-Lindner, Camilla: Die Türkei und Ägypten in der Weltwirtschaftskrise 1929-1933. Stuttgart: Franz Steiner 1989.

Der Handel der Türkei. Balkan-Revue 2 (1915-1916), S. 111-119, 248-261, 419-428.

Der Prozeß Talaat Pascha. Stenographischer Bericht über die Verhandlung gegen den des Mordes an Talaat Pascha angeklagten armenischen Studenten Salomon Teilirian. Mit einem Vorwort von Armin T. Wegner und einem Anhang. Berlin: Deutsche Verlagsgesellschaft für Politik und Geschichte 1921 .

Die neue Türkei 1919-1927. Rede gehalten von Gazi Mustafa Kemal Pascha in Angora vom 15.-20. Oktober 1927 vor den Abgeordneten und Delegierten der Republikanischen Volkspartei. 3 Bde. Leipzig: Koehler 1928.

Fischer, Fritz: Griff nach der Weltmacht. Die Kriegszielpolitik des kaiserlichen Deutschland 1914/18. Düsseldorf: Droste 1967 (Sonderausgabe).

Fischer, Fritz: Krieg der Illusionen. Die deutsche Politik von 1911-1914. 2. Aufl. Düsseldorf: Droste 1970.

Fleury, Antoine: La pénétration allemande au Moyen-Orient 1919-1939: Le cas de la Turquie, de l'Iran et de l'Afghanistan. Genève: A.W. Sijthoff 1977.

Gross, Hermann: Die türkische Republik und Europa. Zeitschrift für Geopolitik II (1934).

Hagen, Gottfried: Die Türkei im Ersten Weltkrieg. Flugblätter und Flugschriften in arabischer, persischer und osmanisch-türkischer Sprache aus einer Sammlung der Universitätsbibliothek Heidelberg. Frankfurt am Main: Peter Lang 1990.

Harbsmeier, Michael: Reisebeschreibungen als mentalitätsgeschichtliche Quellen. Überlegungen zu einer historisch-anthropologischen Untersuchung frühneuzeitlicher deutscher Reisebeschreibungen. In: Reiseberichte als Quellen europäischer Kulturgeschichte. Hrsg. von Antoni Mączak, Hans-Jürgen Teuteberg. Wolfenbüttel: Herzog-August Bibliothek 1982, S. 1-31.

Hartmann, Richard: Ziya Gökalp’s Grundlagen des türkischen Nationalismus. Orientalische Literatur-Zeitung 28 (1925), S. 578-610.

Herlt, Gustav: Die Industrialisierung der Türkei. In: Geld, Industrialisierung und Petroleumschätze der Türkei. Hrsg. von Hugo Grothe et al. Berlin: De Gruyter 1918, S. 41-80.

Hirsch, Ernst E.: Aus des Kaisers Zeiten durch die Weimarer Republik in das Land Atatürks. München: Schweitzer 1982.

Honig, N. von: Über Industrie und Handwerk in Konstantinopel. Archiv für Wirtschaftsforschung im Orient 1 (1916), S. 421-448, 2 (1917), S. 76-108.

Izzet Pascha, Ahmed: Denkwürdigkeiten. Ein kritischer Beitrag zur Kriegsschuldfrage. Hrsg. von Karl Klinghardt. Leipzig: Koehler 1927.

Jäckh, Ernst: Der aufsteigende Halbmond. Beiträge zur türkischen Renaissance. Berlin: Fortschritt (Buchverlag der „Hilfe“) 1909, 5., erw. Aufl. Stuttgart: Deutsche Verlags-Anstalt 1915.

Jäckh, Ernst: Deutschland im Orient nach dem Balkankrieg. München: Mörike 1913.

Jäckh, Ernst: Die deutsch-türkische Waffenbrüderschaft. In: Der Deutsche Krieg. Politische Flugschriften 24. Hrsg. von Ernst Jäckh. Stuttgart: Deutsche Verlags-Anstalt 1915.

Jäschke, Gotthard: Der Weg zur russisch-türkischen Freundschaft. Die Welt des Islams 16 (1934), S. 23-38. 
Jäschke, Gotthard: Die Türkei seit dem Weltkriege. Geschichtskalender, 1918-1928. Berlin: Deutsche Gesellschaft für Islamkunde 1929.

Jäschke, Gotthard: Kommunismus und Islam im türkischen Befreiungskriege. Die Welt des Islams 20 (1938), S. 110-117.

Kaerger, Karl: Kleinasien. Ein deutsches Kolonisationsfeld. Kolonialwirthschaftliche Studie. Berlin: Gergonne 1892.

Kampen, Wilhelm van: Studien zur deutschen Türkeipolitik in der Zeit Wilhelms II. (Diss.) Kiel: Disputats 1968.

Kannengießer, Hans: Gallipoli. Bedeutung und Verlauf der Kämpfe 1915. Berlin: Schlieffen 1927.

Koçak, Cemil: Türkiye'de bir Alman subayı. Nazi Almanya’sının Ankara Ataşemiliteri (1936-44) General H. Rohde'nin Türkiye anıları hakkında oğlu Dr. Dieter Rohde ile bir konuşma. Tarih ve Toplum 5 (Mai 1984), S. 18-27.

Krautheim, Hans-Jobst: Öffentliche Meinung und imperiale Politik. Das britische Rußlandbild 1815-1854. Berlin: Duncker \& Humblot in Kommission 1977.

Lepsius, Johannes: Deutschland und Armenien, 1914-1918. Sammlung diplomatischer Aktenstücke. Potsdam: Tempelverlag 1919.

Lichtenstädter, Siegfried: Nationalität, Religion und Berufsgliederung im Oriente. In: Jahrbuch der Münchner Orientalischen Gesellschaft. Hrsg. von Hugo Grothe. (Beiträge zur Kenntnis des Orients VIII) Halle an der Saale: Gebauer-Schwetschke 1910, S. 42-70.

Marré, Ernst C.N.: Die Türken und Wir nach dem Kriege. Ein praktisches Wirtschaftsprogramm. Berlin: C.A. Schwetschke \& Sohn 1916.

Mejcher, Helmut: Die Bagdadbahn als Instrument deutschen wirtschaftlichen Einflusses im Osmanischen Reich. Geschichte und Gesellschaft 1 (1975), S. 447-481.

Melzig, Herbert: Kamál Atatürk - Untergang und Aufstieg der Türkei. Frankfurt am Main: Societäts-Verlag 1937.

Menz, Reinhold: Deutsche Arbeit in Kleinasien. Reiseskizze und Wirthschaftsstudie. Berlin: Springer 1893.

Mikusch, Dagobert von: Gasi Mustafa Kemal. Leipzig: Paul List 1929.

Morawitz, Karl: Aus Arbeitstagen und Mußestunden. Wien: Braumüller 1907.

Mühlmann, Carl: Der Kampf um die Dardanellen 1915. Oldenburg in Oldenburg: Stalling 1927.

Mühlmann, Carl: Deutschland und die Türkei 1913-1914. Die Berufung der deutschen Militärmission nach der Türkei 1913, das deutsch-türkische Bündnis 1914 und der Eintritt der Türkei in den Weltkrieg. Berlin-Grunewald: Rothschild 1929.

Mygind, Eduard: Anatolien und seine wirtschaftliche Bedeutung. Balkan-Revue 4 (1917-1918), S. $1-6$.

Naumann, Friedrich: „Asia“. Eine Orientreise über Athen, Konstantinopel, Baalbek, Nazareth, Jerusalem, Kairo, Neapel. Berlin: Buchverlag der „Hilfe“ 1899 und 7. Aufl. 1909.

Neumark, Fritz: Zuflucht am Bosporus. Deutsche Gelehrte, Politiker und Künstler in der Emigration 1933-1953. Frankfurt am Main: Knecht 1980.

Oppenheim, Max Freiherr von: Zur Entwickelung des Bagdadbahngebietes und insbesondere Syriens und Mesopotamiens unter Nutzanwendung amerikanischer Erfahrungen. Berlin: Liebheit \& Theisen 1904.

Oppenheim, Max Freiherr von: Die Nachrichtensaal-Organisation und die wirtschaftliche Propaganda in der Türkei, ihre Übernahme durch den Deutschen Überseedienst. Berlin: Reichsdruckerei 1917.

Pomiankowski, Josef: Der Zusammenbruch des ottomanischen Reiches. Erinnerungen an die Türkei aus der Zeit des Weltkrieges. Zürich: Amalthea 1928.

Range, Paul: Vier Jahre Kampf ums Heilige Land. Bad Oeynhausen: Meyer 1932. 


\section{2| Filkret Adanur}

Rühl, Phillip: Die Türkische Republik in Wirtschaft und Aufbau. In: Schriften des Frankfurter Messamts 16) Frankfurt am Main: R. Th. Hauser \& Co. [1925].

Sanders, Liman von: Fünf Jahre Türkei. Berlin: August Scherl 1919.

Schlagintweit, Max: Deutsche Kolonisationsbestrebungen in Kleinasien. Vortrag gehalten in der Deutschen Kolonial-Gesellschaft, Abteilung Berlin, am 8. Januar 1900. München: Wolf \& Sohn 1900.

Schlegel, Dietrich: Das Bild der Türkei in der deutschen Presse 1924-1933. Südosteuropa-Mitteilungen 26 (1986), S. 20-39.

Schöllgen, Gregor: „Dann müssen wir uns aber Mesopotamien sichern!“ Motive deutscher Türkenpolitik zur Zeit Wilhelms II. in zeitgenössischen Darstellungen. Saeculum 32 (1981), S. 130-145.

Schopp, Georg Michael: Türkische Jugend in Deutschland. Ein Beitrag zur Geschichte der deutsch-türkischen Beziehungen zu Beginn des 20. Jahrhunderts. In: Düsseldorfer Jahrbuch 74. Hrsg. von Düsseldorfer Geschichtsverein. Düsseldorf: Klartext 2003.

Schreiber, Gerhard; Stegemann, Bernd; Vogel, Detlef: Der Mittelmeerraum und Südosteuropa. Das Deutsche Reich und der Zweite Weltkrieg 3. Hrsg. vom Militärgeschichtlichen Forschungsamt. Stuttgart: Deutsche Verlagsanstalt 1984.

Schwarzhaupt, Paul: Die Wahrheit über die Türkei. Die Armeniergreuel und Deutschenbedrückungen durch die Jungtürken. (Revolutions-Bibliothek Nr. 6) Berlin: Gesellschaft und Erziehung 1919.

Seiger, Bernhard: Die Mühlenindustrie in und um Konstantinopel. Balkan-Revue 4 (1917-1918), S. 520-528.

Skalweit, Stephan: Frankreich und Friedrich der Große. Bonn: Röhrscheid 1952.

Sullivan, Charles Donald: Stamboul Crossings. German Diplomacy in Turkey, 1908 to 1914. (PhD thesis) Nashville, Tennessee: Vanderbilt University 1977.

Trott-Helge, Else: Die Erweiterung der Tätigkeit der türkischen Landwirtschaftsbank. Balkan-Revue 4 (1917-1918), S. 384-387.

Trumpener, Ulrich: Germany and the Ottoman Empire 1914-1918. Princeton, NJ: Princeton University Press 1968.

Tunçay, Mete: Alman sol basınında Türkiye konuları. Tarih ve Toplum No. 48 (Dezember 1988), S. 6-8; No. 50 (Februar 1988), S. 5-8; No. 55 (Juli 1988), S. 6-9; 49 No. 56 (August 1988), S. 6-8; No. 57 (September 1988), S. 51-52.

Ular (Uhlemann), Alexander; Insabato, Enrico: Der erlöschende Halbmond. Türkische Enthüllungen. Frankfurt am Main: Rütten \& Loening 1909.

Vincenz, Friedrich von: Die türkische Tabakregie. Balkan-Revue 1 (1914-1915), S. 118-126.

Wenger, Klaus Rudolf: Preussen in der öffentlichen Meinung Frankreichs 1815-1870. Politische Aspekte des französischen Preußenbildes. Ein Beitrag zur historischen Analyse nationaler Urteilsklischees. Göttingen: Musterschmidt 1979.

Widmann, Horst: Exil und Bildungshilfe. Die deutschsprachige Emigration in die Türkei nach 1933. Bern: Peter Lang 1973.

Wittek, Paul: Konstantinopel, Islam und Kalifat. Archiv für Sozialwissenschaft und Sozialpolitik 53 (1925), S. 370-426.

Zapp, Manfred: Türkischer Nationalsozialismus. Preußische Jahrbücher (Aug. 1933), S. 105-112.

Ziemke, Kurt: Die neue Türkei - Politische Entwicklung 1914-1929. Berlin, Leipzig: Deutsche Verlags-Anstalt 1930. 